\title{
http://doi.org/10.15359/ree.2003-4.9 \\ PLANEAMIENTO EN EL JARDÍN INFANTIL
}

\author{
Ana Polanco Hernández*
}

El artículo "Laplanificación en el jardin infantil" ofrece algunas estrategias que se pueden considerar para elaborar un planeamiento didáctico destinado a favorecer el desarrollo integral de los niños del nivel inicial.

También se plantean varias opciones para atender al niño (a) integralmente durante toda su etapa preescolar, desde los 0 hasta los 6 años aproximadamente, asi como algunas caracteristicasque el docente de este nivel debe poseer.

Además se exponen los tres momentos que requiere la planificación de aula, a saber, diagnóstico y adaptación, desarrollo y cierre. Por último, se proponen tres modelos de planeamiento, ya sea por tareas de desarrollo, propósitos u objetivos.
This article includes some strategies that can be helpful for didactics planning oriented tofavoring integral development for initial level children. It also considers several options for an integral attention of children for the whole preschool stage, from zero to six years approximately; as well as some teacher characteristics necessany for teaching at this level.

Furthermore, the article discusses three phases required for classroom planning: diagnosis and adaptation, development and closing. Finally, it proposes three planning models, considering development tasks, objectives and goals.

* Es Bachiller y Licenciada en Educación Preescolar, Magíster en Planificación Curricular de la Universidad de Costa Rica. Ha realizado trabajo directo con niños preescolares por 17 años, de los cuales 12 fueron en el ciclo Preescolar de la Escuela Nueva Laboratorio de la Universidad de Costa Rica. Actualmente se desempeña como Profesora en la Escuela de Formación Docente, Facultad de Educación. Universidad de Costa Rica. 


\section{Educación preescolar en Costa Rica}

La educación preescolar en Costa Rica nace en la segunda década del siglo $\mathrm{XX}$, como consecuencia de una necesidad económica y como una respuesta a un movimiento social que incorpora a la mujer a la fuerza laboral del país.

En este contexto social y económico, las instituciones de educación preescolar surgen con el objetivo de atender y asistir a los niños ${ }^{1}$ de las madres trabajadoras; por ello, en 1925, abre las puertas el jardín público Maternal Montesoriano, destinado al trabajo con niños de 5 y 6 años, el cual está ubicado hasta el día de hoy en el centro de la provincia de San José.

En las décadas siguientes, nacieron algunos otros jardines infantiles públicos en el centro de San José, como por ejemplo, Jardín Infantil Justo A. Facio, en Barrio Luján, Margarita Esquivel, en Barrio México; Juan Rafael. en Mora Barrio Don Bosco; así como también en los centros de las provincias de Heredia, Alajuela y Cartago.

En los años 70, el Estado costarricense crea el Programa de Asignaciones Familiares, administrado por el Ministerio de Salud y el Ministerio de Educación Pública, con el fin de mejorar las condiciones de salud y nutrición de los niños en riesgo social. Es así como surgen los Centros Infantiles de Atención Integral (CINAI) y los Centros de Salud y Nutrición (CEN) (Conforme Programa de Bachillerato de la carrera de Educación Preescolar, UCR, 1996).

Simultáneamente se crean centros infantiles adscritos a empresas privadas e instituciones autónomas. Dentro de este grupo podemos señalar al Instituto Nacional de Seguros (INS), el Instituto Nacional de Electricidad (ICE), la Universidad de Costa Rica (UCR), y el periódico La Nación, entre otros.

La iniciativa privada encuentra en la educación infantil un terreno fértil y, como consecuencia, aparecen los jardines infantiles pequeños ${ }^{2}$, ubicados en la mayoría de las comunidades urbanas costarricenses.

Como puede observarse, a partir de la década de los 70 la educación preescolar crece rápidamente; sin embargo, desde ese momento, los programas se caracterizaban por ser fundamentalmente de carácter asistencial, dejando de lado la orientación pedagógica; unido a esto, se carecía de un programa estatal que regulara la atención del niño de 0 a 5 años y seis meses, conocido hoy como el ciclo materno infantil.

No es sino en marzo del año 2000, cuando el Ministerio de Educación Pública ofrece al país una guía o programa de estudio que orienta la labor educativa de este ciclo.

1 En adelante se utilizará el sustantivo niño para designar tanto al género masculino como al femenino. Esto por guardar una concordancia gramaticalmente correcta entre el verbo y sus complementos y por favorecer la lectura ágil del texto.

2 Se designa aquí a todo aquel centro educativo o de cuidado infantil preescolar de infraestructura reducida: una casa de habitación o parte de ella cuyo funcionamiento puede darse dentro o al margen del conocimiento municipal o gubernamental. 
Actualmente vivimos una etapa de transición, pues algunas instituciones. tanto de carácter público como privado, están en proceso de familiarización con el nuevo programa educativo y otras continúan con una línea asistencial.

En vista de lo anterior. el presente artículo trata de ofrecer algunas estrategias que se pueden considerar a la hora de elaborar un planeamiento didáctico que favorezca el desarrollo integral de los niños ubicados en el nivel preescolar.

En primer lugar, se recomienda atender las necesidades e intereses que cada grupo de edad va requiriendo, para ello el docente debe ser un conocedor del desarrollo del niño, con el propósito de que pueda diferenciar y atender las intereses y las necesidades propias de sus alumnos.

\section{Desarrollo del niño.}

De acuerdo con Hernández y Rodríguez (1996, p.:15), entendemos por desarrollo del niño "... un proceso gradual y continuo de cambio en lo referente a la cantidad y calidad de las conductas que el sujeto va presentando durante la vida". Por otra parte, para León (1998) el desarrollo se inicia desde que el óvulo es fertilizado, y nace una vida, la cual experimentará cambios a través de diferentes etapas por las que el individuo pasará hasta concluir con la muerte; siendo la infancia una de estas etapas.

En el caso del niño menor de seis años, el desarrollo implica no solamente cambio fisiológicos, como el aumento en el tamaño de su cuerpo, sino también cambios graduales en la manera de sentir, actuar y pensar; y en ocasiones estas transformaciones no pueden verse a simple vista. Un ejemplo de esto lo constituyen las investigaciones que la neurociencia ha realizado, las cuales demuestran cómo las experiencias tempranas tienen un impacto decisivo en la arquitectura del cerebro y en la naturaleza de las capacidades, que se prolongan en la edad adulta (Corrales, 2000. C.D).

Es importante estar consciente de que cada niño tiene un desarrollo distinto de los otros, pero que cada etapa por la que pasa tiene un orden secuencial. Así por ejemplo, generalmente el niño primero gatea y luego camina; sin embargo, no todos lo logran en el mismo período de tiempo.

En consecuencia, el docente debe ser conocedor de las diferentes etapas de desarrollo por las que el pequeño ${ }^{3}$ irá pasando, así como los márgenes mínimos de tiempo que se han establecido para la ejecución de cada una de ellas, de esta forma estará pendiente del desarrollo integral de niño, y logrará estimularlo y guiarlo en forma adecuada.

3 El término pequeño referido a niño, se utiliza aquí como sustantivo, en su acepción "De muy corta edad". (D.R.A.E. 1992). Se hace la aclaración por interpretaciones diferentes que puedan derivarse de su utilización. 
Con el propósito de comprender, estudiar e investigar el desarrollo del niño. éste se divide en distintas áreas; no obstante, no debe interpretarse ese desarrollo fraccionado, por el contrario, el niño es un ser integral y como tal debe tratarse.

Una de las divisiones más frecuentes en la bibliografía especializada es aquella que plantea como áreas de desarrollo la socioafectiva, la motora gruesa. la motora fina, la cognitiva y, la lingüística, y en el caso de abarcar recién nacidos se suelen incorporar los reflejos como otra área más. A continuación, se define brevemente cada área de desarrollo.

\section{Reflejos}

Area constituida por las respuestas automáticas que se presentan en el recién nacido. Estas conductas del sistema nervioso central son la base de características futuras del niño.

\section{Socioafectiva}

Según Cerdas, Polanco y Rojas (2000, p.: 46), "es un proceso mediante el cual el niño adquiere conductas y construye creencias, normas, actitudes, valores propios del medio cultural y familiar en el que se desenvuelve, para establecer relaciones armoniosas consigo mismo, con los demás y con medio que lo rodea". Según Erikson (1976) estas primeras interacciones sientan las bases para el desarrollo socioemocional.

Cuando se trata de niños pequeños, la seguridad, el afecto y la compañía deben de satisfacerse adecuadamente, para permitir el desarrollo de sentimientos de seguridad y lazos de afectividad.

\section{Motora gruesa}

Esta área se refiere a los grupos musculares grandes que involucran actividades como darse vuelta, gatear o caminar (Cobos (1997). En el jardín infantil el desarrollo de esta área es básico, ya que los niños preescolares requieren de la ejercitación de los músculos gruesos, o sea, las piernas, los brazos o el tronco para que los movimientos se tornen cada vez más precisos.

\section{Motora fina}

Según Cobos (1997) esta se refiere a la actuación de grupos musculares pequeños, principalmente aquellos que controlan los movimientos de los dedos. Para los docentes de educación preescolar esta área es significativa, dado que al ejercitar los músculos finos el niño logra ejecutar movimientos cada vez más precisos, hasta llegar a tomar un lápiz en forma correcta y escribir. 


\section{Cognitiva}

En esta área "se involucra el proceso mediante el cual el niño adquiere conocimientos acerca de sí mismo, de los demás y del medio en el que se desenvuelve" (Cerdas, Polanco y Rojas 2000, p.:57) En el nivel preescolar, esta área se estimula al favorecer la construcción de conocimientos, mediante las interacciones que el niño realiza con los demás, con los objetos y con el medio que lo rodea.

\section{Lingüística}

El proceso de desarrollo del lenguaje juega un papel muy importante, porque abarca las conductas que le permiten al niño comunicarse con las personas que lo rodean. El progreso en esta área es de suma importancia durante la etapa preescolar, ya que por medio de ésta el niño logra expresar sus pensamientos, sentimientos y emociones.

Después de establecer estas breves pero valiosas definiciones, en lo que concierne a la labor docente, queda claro que, para potenciar el desarrollo integral de los niños que reciben educación infantil desde muy temprana edad, deben incorporarse al planeamiento didáctico, en forma equitativa, cada una de las áreas involucradas ene este desarrollo.

\section{Características del docente}

Una concepción pedagógica que potencie el desarrollo integral requiere de un maestro con características personales y profesionales particulares; éstas se encuentran muy bien desarrolladas en el Programa de Materno Infantil (MEP 2000) y en el Programa de Estudios Ciclo de Transición (MEP 1996), a saber:

- Ser coherente entre lo que se piensa y lo que se practica.

- Tener una buena salud física y mental.

- Ser una persona creativa.

- Ser capaz de escuchar y dar apoyo.

- Ser paciente y flexible.

- Tener gran respeto por los deberes y derechos humanos así como la protección del medio ambiente.

- Valorar la cultura autóctona y otras expresiones de la cultura universal.

- Poseer relaciones humanas afectivas, positivas y de respeto mutuo.

- Poseer confianza en sí misma y perseverancia al desarrollo de su trabajo.

- Capaz de adaptarse a diferentes contextos.

- Poseer adecuada disposición para comunicarse, interactuar y realizar trabajos en conjunto con las familias. 
- Mostrar sentido del humor.

- Responsable con su trabajo.

- Mostrar interés de superación personal y profesional.

- Mostrar actitudes de compañerismo.

Respecto a las cualidades profesionales que el docente de educación preescolar debe poseer se citan las siguientes:

- Debe investigar y reflexionar constantemente acerca de su labor pedagógica.

- Participar activamente en la elaboración, ejecución y evaluación de la propuesta curricular.

- Desarrollar acciones a partir de las necesidades e intereses, fortalezas y debilidades de los niños.

- Proveedor de experiencias significativas y creativas.

- Capacidad de entrevistar a niños y adultos.

- Participar en procesos de actualización.

- Trabajar junto con las familias y la comunidad.

- Poseer capacidad de aplicar los conocimientos a situaciones concretas.

- Formación universitaria en educación preescolar.

Por otra parte, la carrera de Bachillerato 'en Educación Preescolar de la Universidad de Costa Rica plantea en su programa de Estudios (1996) que un docente que atiende niños de cero a seis años debe también cumplir con los siguientes papeles y funciones:

- Ser un asistente del desarrollo del niño lo que implica diagnosticar sus necesidades e intereses, favorecer la construcción personal y social, conocer y evaluar el desarrollo del pequeño.

- Ser un integrador social, de la familia y la comunidad así como del entorno físico biológico, social.

- Ser un planificador curricular, que construya y desarrolle un plan curricular de desarrollo integral.

\section{Planeamiento en la educación preescolar}

Una orientación pedagógica que potencie el desarrollo integral del niño requiere tanto de una planificación institucional como de un planeamiento para el trabajo de aula. Con respecto a éste último, debe contemplar tres momentos que marcan el ciclo lectivo, a saber: diagnóstico y adaptación, desarrollo y cierre. 
Diagnóstico y adaptación. Corresponde al inicio del período lectivo y es el momento en el cual el docente recoge la información sobre el niño y su medio. social. familiar, personal, y la analiza para incorporarla a su programación curricular. Para tal efecto, sigue una serie de procedimientos, por ejemplo: entrevistas a los padres de familia o encargados del niño. visitas a la comunidad y a las familias, administración de instrumentos que valoren las diferentes áreas de desarrollo, entre otros.

Por otro lado, también durante este período se trabaja con el niño para conocer sus actitudes, hábitos. habilidades y conocimientos. De esta manera el maestro conoce el perfil real de sus alumnos y a partir de este organiza el trabajo de la siguiente etapa.

Otros aspectos por considerar durante esta etapa de diagnóstico son: las características del personal docente y administrativo, los recursos físicos y materiales con que cuenta el jardín infantil, el número de horas que los niños permanecen en la institución, el clima de la región, etc.

No debe olvidarse la importancia que tiene el realizar un análisis de la comunidad en la cual está inmerso el centro educativo y de la que probablemente procedan los niños que forman el grupo, conocer los recursos con que cuenta, así como las características físicas y sociales que presenta, con el fin de contextualizar y dar pertinencia cultural a las experiencias de aprendizaje.

Así mismo, debe tenerse en cuenta que al iniciar el ciclo lectivo los niños y sus familias se encuentran en período de adaptación, lo que podría generar un ambiente ansioso, tenso o de angustia, de ahí la importancia de establecer un acercamiento armonioso, de confianza entre la familia, los adultos que estarán a cargo de los niños y la institución a nivel general.

Con respecto a la adaptación de los niños, debe tenerse presente que durante esta etapa del ciclo lectivo el niño tiene sus primeros contactos con el jardín infantil, sus miembros y sus dependencias, lo que hace necesario que también a lo largo de este período descubra este grupo social, experimente las variadas relaciones y situaciones que en él se dan y se adapte a la vida en grupo.

Desarrollo. Este segundo momento se inicia cuando el maestro considera que ha logrado la integración que esperaba durante el período de adaptación, y se extiende hasta unas semanas antes de concluir el ciclo lectivo. Durante este lapso, se deben seleccionar, organizar y relacionar los componentes didácticos con los que se trabajará la mayor parte del curso lectivo, hasta que se inicie el último período.

Cierre Para Denies (1992, p.: 149), este período abarca el tiempo que corresponde al cierre del ciclo lectivo y dependerá de las características del grupo, de la institución y la comunidad. El propósito de este momento es la integración y evaluación de los procesos y resultados de los aprendizajes desarrollados durante el año, así como la programación de los diferentes elementos curriculares (objetivos, contenidos, actividades y recursos). 
Enfoques curriculares utilizados para el planeamiento didáctico

Indistintamente del período lectivo en el que se trabaje, el proceso de planeamiento es indispensable, dado que planear implica tomar las previsiones necesarias para alcanzar un determinado objetivo. lograr una meta o cumplir una misión. El planeamiento que cada maestro realiza es muy personal, ya que depende de las características del grupo de niños, de él mismo como docente, y del enfoque curricular que se utilice.

Así por ejemplo, si se atiende un grupo de niños menores de tres años, utilizar tareas de desarrollo es una buena opción. dado que este grupo en particular presenta una serie de cambios progresivos muy rápidos en su desarrollo.

Ahora bien, se entiende por tareas de desarrollo "aquellas que enuncian un aspecto clave e integrador del desarrollo, que toda persona debería poseer o superar satisfactoriamente en ciertos períodos de la vida, respondiendo así a sus propias necesidades, como a las de la cultura en la que participa, un ejemplo de ellas puede ser "aprendizaje del lenguaje". Peralta (1996, p.: 106)

Sin embargo, muchos docentes planean mediante propósitos, los cuales, según la misma autora corresponden a una acción general del educador; estos se plantean en infinitivo, poseen un contenido, un aspecto del desarrollo o efecto de esa acción. Los propósitos son conceptualizados como un proceso permanente de interacción entre las características maduracionales de desarrollo del niño, y el medio social, cultural, físico y natural que lo rodea.(Programa Materno Infantil, MEP 2000).

Algunos docentes planean mediante objetivos, los cuales según Denies (1992) son metas amplias o precisas que orientan la programación y práctica de la labor de aula.

\section{Modelos de planeamiento para el jardín infantil}

A continuación se presenta una propuesta para planear con tareas de desarrollo, otra con propósitos y una con objetivos. Los elementos curriculares que se contemplan son aquellos que la autora considera indispensables; no obstante, es factible agregar otros, como por ejemplo: una columna de materiales y otra de valores anexos con juegos, canciones, propuestas de evaluación, etc. 
Modelo 1. Planeamiento por tareas de desarrollo.

MAESTRA:

Edad del niño:

1- TAREA (S) DE DESARROLLO: Área a la que corresponde. Ejemplo:

1. Reflejos

2. Motora gruesa

3. Motora fina

4. Cognitiva

5. Lingüística

6. Socioafectiva

\begin{tabular}{|l|l|l|}
\hline \multicolumn{1}{|c|}{$\begin{array}{c}\text { SUBTAREA DE } \\
\text { DESARROLLO }\end{array}$} & \multicolumn{1}{c|}{ ACTIVIDADES } & \multicolumn{1}{c|}{ EVALUACIÓN } \\
\hline $\begin{array}{l}\text { 1. Primera subtarea de } \\
\text { desarrollo que } \\
\text { corresponde al área } \\
\text { motora gruesa. }\end{array}$ & $\begin{array}{l}\text { 1.1. Primera actividad de } \\
\text { la subtarea de desarrollo 1. } \\
\text { 1.2. Segunda actividad de } \\
\text { la subtarea de desarrollo } \\
\text { 1.3. Tercera actividad de } \\
\text { lasubtarea de desarrollo 1. }\end{array}$ & $\begin{array}{l}\text { 1.1. Evaluación } \\
\text { correspondiente a la } \\
\text { subtarea de desarrollo 1. } \\
\text { Instrumento que se } \\
\text { utilizará. }\end{array}$ \\
$\begin{array}{l}\text { 2. Segunda subtarea } \\
\text { de desarrollo motor } \\
\text { grueso (en caso de } \\
\text { existir) }\end{array}$ & $\begin{array}{l}\text { 2.1. Primera actividad de la } \\
\text { subtarea de desarrollo 2. } \\
\text { 2.2. Segunda actividad de } \\
\text { la subtarea de desarrollo 2. } \\
\text { 2.3.Tercera actividad de la } \\
\text { subtarea de desarrollo 2. }\end{array}$ & $\begin{array}{l}\text { 2.1. Evaluación } \\
\text { Correspondiente a la } \\
\text { subtarea de desarrollo 2. } \\
\text { Instrumento que se }\end{array}$ \\
$\begin{array}{l}\text { utilizará. } \\
\text { 3. Tercera subtarea de } \\
\text { desarrollo que } \\
\text { corresponde al área } \\
\text { cognitiva. }\end{array}$ & $\begin{array}{l}\text { 3.1. Primera actividad de } \\
\text { la subtarea de desarrollo 3. } \\
\text { 3.2. Segunda actividad de } \\
\text { la subtarea de desarrollo 3. } \\
\text { 3.3.Tercera actividad. De } \\
\text { la subtarea de desarrollo 3. }\end{array}$ & $\begin{array}{l}\text { 3.1. Evaluación } \\
\text { correspondiente a la } \\
\text { subtarea de desarrollo 3. } \\
\text { Instrumento que se } \\
\text { utilizará. }\end{array}$ \\
\hline
\end{tabular}




\section{2- FUNCIONES O APORTES DE LA FAMILIA:}

\section{3- BIBLIOGRAFÍA UTILIZADA}

Para el siguiente ejemplo, correspondiente al planeamiento por propósitos. se utilizan los propósitos del Programa de Materno infantil (MEP 2000), a saber:

Que la niña y el niño:

Se adapten progresivamente al entorno sociocultural al que pertenece según las normas, valores, costumbres y tradiciones del medio de acuerdo con su nivel de desarrollo.

Construyan su autonomía e identidad personal a partir de diversas interacciones con el medio que les rodea.

Amplíen y profundicen paulatinamente sus experiencias y conocimientos al interactuar con el ambiente a partir del nivel de desarrollo en que se encuentran.

Enriquezcan y diversifiquen sus formas de comunicación, expresión y representación de la realidad en interacción con el contexto sociocultural.

\section{Modelo 2. Planeamiento por propósitos}

\section{MAESTRA:}

\section{Edad del niño:}

1- Propósito (s) del programa de Materno Infantil:

\begin{tabular}{|l|l|l|}
\hline \multicolumn{1}{|c|}{$\begin{array}{c}\text { PROPÓSITOS } \\
\text { ESPECÍFICOS }\end{array}$} & \multicolumn{1}{c|}{$\begin{array}{c}\text { ACCIONES Y } \\
\text { ACTIVIDADES }\end{array}$} & \multicolumn{1}{|c|}{ EVALUACIÓN } \\
\hline $\begin{array}{l}\text { Primer propósito } \\
\text { específico que } \\
\text { corresponde al } \\
\text { propósito } \\
\text { seleccionado. }\end{array}$ & $\begin{array}{l}\text { Acción (es) que se } \\
\text { proponen para el } \\
\text { propósito. Actividades } \\
\text { que se emanan de la } \\
\text { acción propuesta. }\end{array}$ & $\begin{array}{l}\text { Evaluación } \\
\text { correspondiente al } \\
\text { propósito específico. } \\
\text { Instrumento que se } \\
\text { utilizará. }\end{array}$ \\
\hline
\end{tabular}




\begin{tabular}{|l|l|l|}
\hline \multicolumn{1}{|c|}{$\begin{array}{c}\text { PROPÓSITOS } \\
\text { ESPECÍFICOS }\end{array}$} & \multicolumn{1}{c|}{$\begin{array}{c}\text { ACCIONES Y } \\
\text { ACTIVIDADES }\end{array}$} & EVALUACIÓN \\
\hline $\begin{array}{l}\text { Segundo propósito } \\
\text { específico } \\
\text { correspondiente al } \\
\text { propósito seleccionado. }\end{array}$ & $\begin{array}{l}\text { Acción o acciones que } \\
\text { se proponen para el } \\
\text { segundo objetivo } \\
\text { específico. }\end{array}$ & $\begin{array}{l}\text { Evaluación } \\
\text { correspondiente al } \\
\text { propósito específico 2. }\end{array}$ \\
\hline
\end{tabular}

\section{2- FUNCIONES O APORTES DE LA FAMILIA.}

\section{3- BIBLIOGRAFÍA UTILIZADA}

La tercera opción de planeamiento está destinada para el ciclo de transición, y se basada en el programa oficial del Ministerio de Educación Pública, para este ciclo.

Es importante clarificar que en esta opción se presentan procedimientos y no actividades, pero no deben de confundirse, como suele suceder en nuestro medio. Los procedimientos son definidos en el Programa de Estudios del Ciclo de Transición como "las acciones y condiciones que el alumno realiza para lograr el objetivo, no obstante le corresponde al docente organizar las actividades necesarias para la ejecución."(1996, p.:19)

Al modelo presentado se le pueden adjuntar otras columnas, como pueden ser la de contenidos, la de actividades, la de valores y actitudes. 
Modelo 3. Planeamiento por objetivos

Ciclo de transición

\section{MAESTRA:}

1- OBJETIVO GENERAL: (nace de un bloque temático del programa.)

\begin{tabular}{|l|l|l|}
\hline \multicolumn{1}{|c|}{$\begin{array}{c}\text { OBJETIVO } \\
\text { ESPECÍFICO }\end{array}$} & PROCEDIMIENTOS & \multicolumn{1}{|c|}{ EVALUACIÓN } \\
\hline $\begin{array}{l}\text { Primer objetivo } \\
\text { específico, emanado } \\
\text { del primer objetivo } \\
\text { general. }\end{array}$ & $\begin{array}{l}\text { Procedimiento (s) } \\
\text { propuesto (s) } \\
\text { correspondientes al } \\
\text { primer objetivo } \\
\text { específico. }\end{array}$ & $\begin{array}{l}\text { Propuesta de } \\
\text { evaluación } \\
\text { correspondiente al } \\
\text { primer objetivo. Es } \\
\text { bueno anotar el } \\
\text { instrumento } \\
\text { evaluativo que se } \\
\text { utilizará. }\end{array}$ \\
\hline
\end{tabular}

\section{2- FUNCIONES O APORTES DE LA FAMILIA.}

\section{3- BIBLIOGRAFÍA UTILIZADA}

\section{Otros elementos a considerar en el planeamiento didáctico}

Además de seleccionar el enfoque curricular, el docente puede incluir en el planeamiento didáctico elementos que considere de importancia.

Un elemento que se propone en todos los posibles modelos de planeamiento y que se incluye en los modelos aquí sugeridos es la evaluación, la cual según Peralta (1996.: p 146) es "Un proceso constante, dinámico, que pretende la objetividad a través del cual se emite un juicio valorativo que ayuda a la determinación de nuevas alternativas de decisión en relación a los diferentes agentes y elementos del currículo"

Es importante hacer énfasis en algunos puntos mencionados en esta definición como la objetividad, la que debe tratar de alcanzarse en la medida de lo 
posible; de suma importancia también el considerar la evaluación como un proceso continuo, es decir, que en todo momento nunca en un único momento se lleva a cabo. Tampoco hay que olvidar que el propósito de la evaluación es ofrecer pautas que indican los cambios que deben realizarse en la siguiente etapa de la planificación, de acuerdo con los aprendizajes y conductas mostrados por los alumnos.

Independientemente del tipo de planeamiento que se realice, tiene que darse una comunicación constante y un vínculo que debe mantenerse con las familias, dado que éstas son las formadoras de sus hijos durante los primeros años de vida. Las acciones que tanto padres como docentes asuman contribuirán en el desarrollo integral del niño, por esto es conveniente anotar dentro del planeamiento las funciones o aportes específicos que realizará la familia.

Finalmente, no menos relevante es la relación y coherencia que debe darse entre los diferentes elementos curriculares que el docente opte por emplear.

En cuanto al tiempo, el maestro decide el lapso para el cual el planeamiento estará destinado. Las opciones que generalmente se emplean son por mes, por quincena o por semana; sin embargo, se considera un buen tiempo la quincena, pues el mes puede convertirse en un período largo y difícil de manejar con niños pequeños, debido a que por sus características de desarrollo suelen perder el interés sobre un determinado aspecto o bien pueden darse cambios en las actitudes y habilidades que el docente no esperaba. Por el contrario, una semana suele ofrecer poco espacio para llevar a cabo procesos que requieren mayor tiempo y en ocasiones es difícil para el maestro dedicar cada semana un espacio para elaborar con cuidado el planeamiento.

Con el objetivo de organizar el planeamiento suele utilizarse una minuta diaria, la cual posee características que cada docente debe considerar, como el tiempo que el niño estará en el jardín infantil, ya sea todo el día o solamente parte de éste; el nivel de concentración, horarios previamente establecidos por la administración, cantidad de niños, horarios de comida y aseo. Además el Programa de Materno Infantil (MEP 2000) propone ofrecer de forma alternativa:

- Experiencias espontáneas y estructuradas.

- Experiencias colectivas, en pequeños grupos, e individuales.

- Experiencias en espacios interiores y exteriores.

- Experiencias iniciadas por los niños y el docente.

- Experiencias cotidianas e innovadoras.

Independientemente de estas situaciones, deben respetarse algunas características que son importantes en la minuta, por ejemplo, cuanto más pequeño 
sea el niño los periodos de alimentación, sueño y aseo ocupan mayor cantidad de tiempo, y en ocasiones es necesario que se den varias veces durante el día: en tanto que los niños más grandes pueden elegir y proponer mayor cantidad de actividades para llevar a cabo.

Por último, pero no por ello de menor importancia, hay que recordar que la minuta es una propuesta diaria y que puede sufrir cambios inesperados, dado que se trata de una proposición de trabajo flexible que debe acomodarse a las circunstancias. De igual forma, es probable que al avanzar el ciclo lectivo ésta sufra transformaciones, pues las características del grupo a nivel general también cambian durante el año.

\section{A manera de conclusión}

En conclusión, puede apreciarse que, como se señala al inicio de este artículo, la educación preescolar ha sufrido transformaciones como consecuencia de los cambios y demandas sociales. Así su objetivo al inicio era meramente asistencial, y no era necesaria su atención por parte de un profesional del desarrollo del niño; no obstante, con el transcurrir del tiempo se ha convertido en una tarea destinada a la atención prioritaria de las necesidades e intereses de los pequeños y a estar en manos de un docente con carrera universitaria.

Por otra parte, se han incorporado a los centros infantiles niños cada vez más pequeños, lo cual exige una planificación adecuada a las características de cada grupo de edad. Si este planeamiento didáctico parte de un diagnóstico del niño, de su familia y de su comunidad, y con esta información se plantean las tareas de desarrollo, los propósitos u objetivos por alcanzar durante el ciclo lectivo, se tendrá mayor seguridad de haber satisfecho las necesidades e intereses de los niños, y de haber contribuido con lo que Peralta (1996) denomina desarrollo armonioso de los pequeños.

Los modelos de planeamiento incluidos en este artículo buscan incorporar los elementos que permitan que ese desarrollo integral (asistencial - didáctico) que tan necesario es hoy día

Como profesionales en el campo de la educación preescolar y forjadores del futuro, corresponde a los maestros estar siempre atentos a las nuevas investigaciones en el campo del desarrollo humano, así como de las demandas que la sociedad impone, para construir un mejor futuro en el cual los niños de hoy vivirán mañana. 


\section{Referencias}

Cerdas, Polanco y Rojas. (2000). El proceso educativo de los niños del Grupo Interactivo II: Guía Pedagógica. Tesis de Maestría. Universidad de Costa Rica

Cobos, P. (1997). El desarrollo motor y sus alteraciones. Manual práctico para evaluarlo y favorecerlo. Madrid, España: Ediciones Pirámide.

Corrales , G. (2000). Exploremos el cerebro infantil la conformación de los circuitos neoronales momentos críticos. En: Memoria IV Simposio Educación Preescolar. (2000): CD Rom.

Denies, C. (1992) Didáctica del nivel inicial. Buenos Aires. Argentina: Editorial El Ateneo.

Erikson, E (1976). Infancia y sociedad. Buenos Aires. Argentina: Editorial Homé.

Hernández R. y Rodríguez S. (1996). Manual operativo para la evaluación y estimulación del crecimiento y desarrollo del niño. San José Costa Rica: Editorial Universidad Estatal a Distancia.

León, A. T. (1998). "El maestro y los niños. La humanización del aula". San José. Costa Rica: Editorial de la Universidad de Costa Rica.

Ministerio de Educación Pública. (1996) Programa de Estudios. Ciclo de Transición. Educación Preescolar. San José, Costa Rica.

Ministerio de Educación Pública. (2000). Programa de Estudio. Ciclo Matemo Infantil. San José Costa Rica.

Peralta, V. (1996). El Currículo en el Jardín de Infantes. (Un análisis crítico) Santiago. Chile: Editorial Andrés Bello.

Universidad de Costa Rica. Sección de Preescolar. (1996). Plan de Estudios Bachillerato en Educación Preescolar. San José, Costa Rica. 